3. Tetrodon porphyreus, Schleg.

Tetraodon porphyreus, Schleg. Faun. Jap., Poiss. p. 282, pl. 121. fig. 1 (1850).

Liosaccus porphyreus, Günth. Cat. Fishes, viii. p. 287 (1870).

$a, b, c$. Gensan, August 18th, 1880 .

One fairly adult, two very young specimens; in spirit but in bad condition.

4. Ostracion cubicus, Linn.

Ostracion cubicus, Linn. S. N. i. p. 410 (1766).

Ostracion immaculatus, Schleg. Faun. Jap., Poiss. p. 296 (1850).

a. Gensan, August 16th, 1880.

A single dried specimen.

\title{
2. Liste des Oiseaux recueillis en Corée par M. Jean
} Kalinowski. Par M. L. Taczanowski, C.M.Z.S.

[Received October 4, 1887.]

La collection est recueillie depuis le mois de septembre 1885 jusqu'à la moitié de mars de 1887 , principalement dans les environs de la capitale du pays, jusqu'à Gouran vers le nord.

Selon la relation du collecteur le pays est très pauvre en oiseaux, dépourvu en général de forêts, pauvre en végétation herbacée et très pauvre en eaux continentales. Les moyens de locomotion sont très difficiles; la population partout brutale, inhospitalière, paresseuse et très pauvre, incapable à aider en rien au voyageur. En outre de ces inconvénients le voyageur a eu un obstacle grave dans ses travaux en été de l'année passée lorsque l'épidémie de choléra faisait des grands ravages pendant plus de trois mois dans la population de la capitale et des environs. Quelques centaines d'indigènes succombaient par jour, on n'enterrait que la moitié, tandis qu'on rejettait l'autre moitié de cadavres dans les champs autour de la ville, tous les environs étaient donc empestés à tel point qu'il était impossible de sortir dehors.

Il n'y a donc rien d'étonnant que cette collection est aussi pauvre en espèces. Selon la relation de M. Kalinowski le quart des espèces fournies reste pour nicher dans le pays, tandis que les autres trois quarts n'y sont que de passage.

Le grande pluralité de ces espèces est composée d'oiseaux sibériens, les autres appartiennent à la faune japonaise, dont les trois espèces suivantes, Parus varius, Mecistura trivirgata et Microscelis amaurotis, sont trouvées pour la première fois sur le continent asiatique; les autres espèces sont chinoises ou indiennes, et qui n'ont pas été retrouvées en Sibérie ni dans le pays Oussourien.

Dans une lettre que je viens de recevoir le voyageur communique une liste d'espèces recueillies après l'expédition de cet envoi, dans litquelle il indique plusieurs qui manquaient dans sa première collection; nous pouvons donc espérer qu'il trouvera encore un bon nombre jusqu’à son départ, qui ne va pas tarder. 
L'exploration de M. Kalinowski ne donnera qu'une faible représentation de l'ornis du pays. Elle est très courte et les contrées boisées ne sont pas touchées.

1. Milvus melanotis, Temm. et Schl.

Un mâle et deux femelles tués à Séoul en novembre et décembre. Dimensious :-

0 ad. Long. totale 665 , vol. 1620, aile 525 , queue 343 , bec $48 \mathrm{~mm}$.

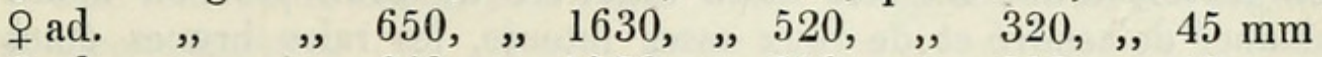
申 ad. " , 640, , 1556, , $510, \quad, 313$, , $45 \mathrm{~mm}$.

\section{Buteo plumipes, Hodgs.}

Un mâle tué en mars.

\section{Astur cuculoides (Temm.).}

Deux mâles et une femelle tués en mai et en juin.

Un de ces mâles est d'un blanc presque pur sur tout le dessous du corps, à plumes de la poitrine d'un vineux pale couvert par le blanc terminal; les côtés colorés par une légère teinte vineuse, les plumes des côtés du bas ventre grises maculées de blanc; le haut des pantalons moucheté de gris; les plumes du milieu de l'abdomen rayées en travers de gris couvert presque complétement par le blanc terminal ; le cendré des parties supérieures du corps est fort bleuâtre et pur. Rectrices, outre les deux médianes, traversées de 5 raies noires, sur la barbe interne des externes il y en a 7 , plus fines et moins complètes que sur les autres. Iris brun foncé, cire et pieds d'un jaune orangé.

Un autre mâle, probablement moins adulte, a le roussâtre plus répandu sur la poitrine, et qui s'étend aussi sur le haut de l'abdomen ; la couleur des parties supérieures du corps moins claire. Iris brun foncé.

La femelle adulte est plus foncée en dessus que le mâle précédent ; gorge largement d'un blane coloré de gris roussâtre et varié de baguettes noires dans toutes les plumes; tout le dessous du corps est d'un roussâtre sâle, presque uniforme sur les côtés et varié de raies transversales sur le milieu de l'abdomen; souscaudales blanches colorées d'une nuance de crème dans la partie terminale des plumes; toute la surface des pantalons fort colorée et mouchetée de gris et de roussâtre ; sousalaires plus fortement roussâtres que celles du mâle, et variées de quelques petites macules noirâtres. Les bandes caudales plus larges que celles du mâle. Iris jaune tirant en orangé ; pieds et cire jaune.

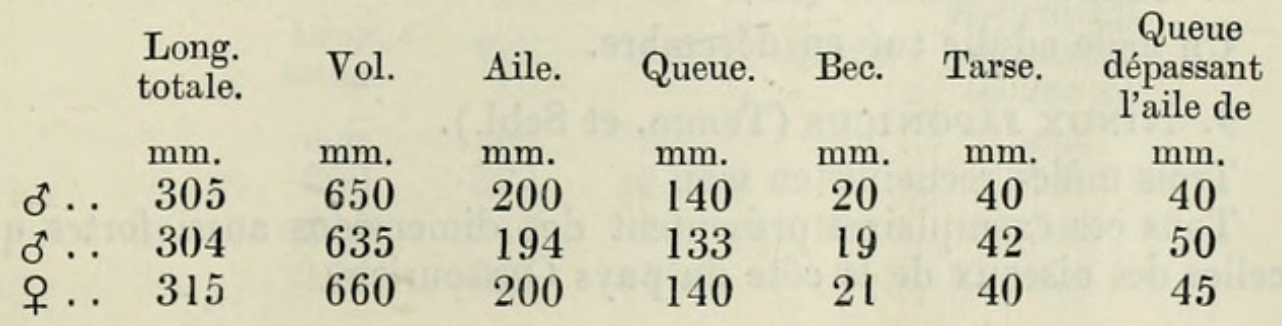




\section{Accipiter nisus (L.).}

Deux mâles et deux femelles tuées en décembre, janvier, février et mars.

\section{Falco hendersoni, Hume.}

Un mâle très adulte tué le 6 janvier 1887 .

Cet oiseau a le sommet de la tête et la nuque roux varié de stries noires centrales dans les plumes; dos et les scapulaires rayés en travers d'une manière assez régulière de brun plus ou moins nuancé de cendré et de roux assez intense, les raies brunes étant plus larges que ces dernières; sur le dos inférieur, le croupion et les tectrices supérieures de la queue le brun est fort nuancé de cendré, les raies rousses remplacées par le cendré clair, à plumes bordées de roussâtre à l'extrémité et la baguette noire, les raies claires des souscaudales externes blanches. Tout le dessous est blanc à l'exception du milieu du ventre et des souscaudales, qui sont d'un blanc de crème; les moustaches fines mais bien prononcées, des petites macules brunes sur les côtés de la tête et du cou, distinctement plus grosses et peu nombreuses sur la poitrine; assez grosses et subarrondies sur l'abdomen; les plus grosses et prolongées en travers sur les côtés du ventre; pantalons fort tachetés. Rectrices rayées en travers de brun et de fauve roussâtre, ces raies complètes dans toutes les pennes au nombre de 14 de chaque couleur. Iris presque noir.

Longueur totale 510 , vol 1160 , aile 375 , queue 210 , queue dépassant l'aile de $40 \mathrm{~mm}$.

6. Dendrofalco exalon (L.).

Un jeune mâle et une femelle, tués en décembre et en février.

7. Cerchneis tinnunculus Japonicus (Temm. et Schl.).

Trois mâles adultes et un jeune mâle, tués en janvier, mars, juin et juillet.

Tous ces exemplaires présentent les caractères de cette race bien prononcés.

\begin{tabular}{|c|c|c|c|c|c|}
\hline $\begin{array}{l}\text { Long. } \\
\text { totale. }\end{array}$ & Vol. & Aile. & Queue. & Tarse. & $\begin{array}{c}\text { Queue } \\
\text { dépassant } \\
\text { l'aile de }\end{array}$ \\
\hline $\begin{array}{r}\mathrm{mm} . \\
360\end{array}$ & $\frac{\mathrm{mm}}{740}$ & $\begin{array}{l}\mathrm{mm} . \\
245\end{array}$ & $\begin{array}{l}\mathrm{mm} . \\
170\end{array}$ & $\begin{array}{c}\mathrm{mm} . \\
38\end{array}$ & $\underset{46}{\mathrm{~mm}}$ \\
\hline 343 & 746 & 240 & 157 & 38 & 38 \\
\hline .334 & 735 & 240 & 154 & 38 & 38 \\
\hline jun. . 360 & 745 & 243 & 153 & 37 & 42 \\
\hline
\end{tabular}

8. Circus cyaneus (L.).

Un mâle adulte tué en décembre.

9. Ninox Japonicus (Temm. et Schl.).

Trois mâles recueillis en mai.

Tous ces exemplaires présentent des dimensions aussi fortes que celles des oiseaux de la côte du pays Oussourien. 


$\begin{array}{cccccc}\begin{array}{c}\text { Long. } \\ \text { totale. }\end{array} & \text { Vol. } & \text { Aile. } & \text { Queue. } & \text { Tarse. } & \begin{array}{c}\text { Queue } \\ \text { dépassant } \\ \text { l'aile de } \\ \mathrm{mm} .\end{array} \\ \mathrm{mm} . & \mathrm{mm} . & \mathrm{mm} . & \mathrm{mm} . & \mathrm{mm} . & \mathrm{mm} . \\ 290 & 783 & 235 & 124 & 27 & 3 \\ 290 & 775 & 227 & 126 & 27 & 7 \\ 293 & 750 & 224 & 125 & 27 & 15\end{array}$

10. Scops semitorques, Temm. et Schl.

Une femelle de Séoul, tuée en février. Iris jaune avec une légère nuance rougeâtre.

11. Bubo ignavus, Forst.

Une femelle adulte, tuée à Séoul en janvier. Iris orangé.

Long. totale 660 , vol 1710 , aile 474 , queue $270 \mathrm{~mm}$.

Ce Duc est en général plus obscur que le mâle de Sidemi, il a les flammules et les taches noires en général plus grosses, les ondulations foncées en général plus larges. Il est aussi en général plus obscur que la grande pluralité des grands Ducs de l'Europe centrale.

12. Syrnium nivicolum, Blyth.

Une paire de Siongnio, recueillie en mars. Phase grise, sans rien de roussâtre et de fauve.

o. Long. totale 420 , vol 940 , aile 276 , queue 180 , tarse 40 , queue dépassant l'aile $40 \mathrm{~mm}$.

ㅇ. Long. totale 445 , vol 1015 , aile 305 , queue 185 , tarse 40 , queue dépassant l'aile $45 \mathrm{~mm}$.

La différence principale du S. aluco est dans les deux rectrices médianes, qui sont distinctement rayées de blanchâtre en travers, il y a 3 raies chez le of sur la partie terminale de la queue, 6 sur les rectrices de la femelle; sur les autres rectrices les raies sont plus blanches; les rémiges primaires terminées par une large bordure blanche ; le dessin des scapulaires un peu plus compliqué, mais ce qui ne produit pas une grande différence; les taches sur la barbe externe des rémiges primaires sont plus blanches; le plumage des pieds plus fortement rayé ou maculé, mais on trouve cependant entre les oiseaux européens des individus à pieds presque aussi fortement tachetés.

13. Caprimulgus jotaka, Temm. et Schl.

Une femelle de Séoul, prise en mai. Iris brun foncé.

14. Cecropis daurica (Pall.).

Trois exemplaires de Séoul, recueillis le 10 juin. Identiques en tout aux oiseaux da la Daourie, à bande rousse uropygiale, large de 24-26 mm.

\begin{tabular}{|c|c|c|c|c|}
\hline $\begin{array}{l}\text { Long. } \\
\text { totale. }\end{array}$ & Vol. & Aile. & Queue. & $\begin{array}{c}\text { Rectrice ext } \\
\text { dépassant } \\
\text { la } 2 \text { me de }\end{array}$ \\
\hline $\begin{array}{l}\mathrm{mm} . \\
200\end{array}$ & $\begin{array}{l}\mathrm{mm} . \\
324\end{array}$ & $\mathrm{~mm}$. & $\mathrm{mm}$. & $\mathrm{mm}$. \\
\hline 210 & 347 & 122 & 106 & 34 \\
\hline 196 & 325 & 114 & 95 & 25 \\
\hline
\end{tabular}




\section{Hirundo gutturalis, Scop.}

Six exemplaires des environs de Seoul, à blanc du dessous du corps presque pur ou coloré d'une très légère nuance roussâtre, comme dans les oiseaux qui nichent dans l'Europe centrale, considérablement moins roux que dans les hirondelles des environs de Wladiwostok et des autres localités du pays Oussourien. Entre les nombreux exemplaires que j'ai vus de ce pays, il n'y a eu qu'un fourni par M. Jankowski, de Sidemi, qui a tout l'abdomen aussi blanc. Dans ces six exemplaires il n'y a que trois dont le roux du devant du cou pénètre profondement dans le noir du collier, ne laissant qu'une fine bordure noire; les trois autres ont la bande noire complète comme celle de l'hirondelle de cheminée européenne. Il ne reste donc dans ces exemplaires d'autres caractères différentiels constants de ceux de l'hirondelle européenne qu'une taille distinctement moins forte, les rectrices externes moins prolongées et la tache blanche aux rectrices plus étroite. Deux de ces mâles ont une strie noire sur les tectrices souscaudales postérieures.

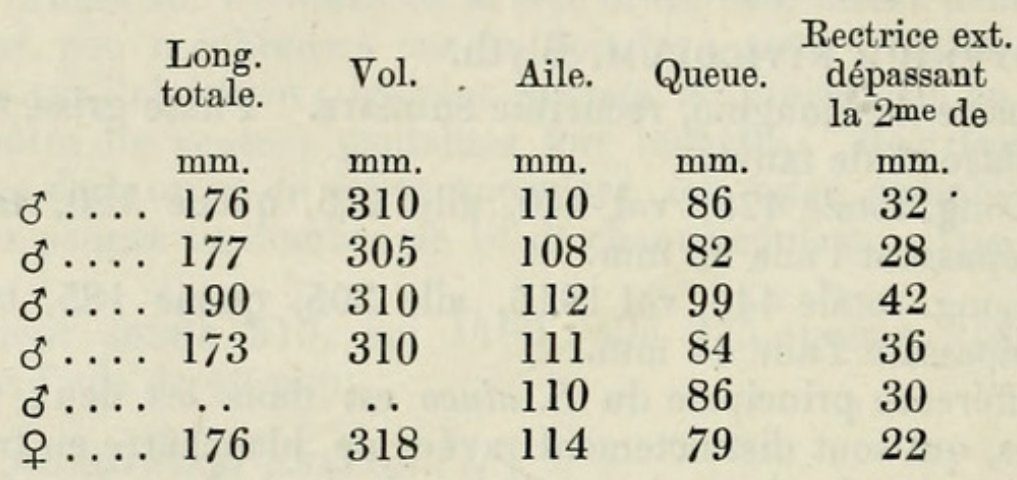

16. Eurystomus orientalis (L.).

Une paire de Séoul, recueillée en juin et juillet.

17. Halcyon pileatus (Bodd.).

Deux mâles et un jeune de Séoul, pris en juin et juillet. Iris brun foncé, bec et pieds rouges.

18. IsPida Bengalensis, Briss.

Deux mâles et une femelle, recueillis à Séoul en avril, mai et juillet. Iris brun foncé.

19. Ceryle lugubris, Temm.

Un mâle d'Ara-Sambo, pris le 1 décembre 1886. Iris brun foncé.

\section{Certhia familiaris, $L$.}

Un exemplaire de Séoul. Cet oiseau ressemble en tout par sa coloration aux oiseaux du Baical méridional, de la Daourie et du pays Oussourien, avec lesquels je l'ai comparé, il a le blanc du dessous également pur, le bec aussi court. que la pluralité de ces oiseaux ; il est cependant d'une taille un peu plus petite, l'aile pliée n'a que $59 \mathrm{~mm}$., tandis qu'un oiseau de l'embouchure de l'Oussouri 
l'a de $63 \mathrm{~mm}$., un de Koultouk $62 \mathrm{~mm}$., une paire de la Daourie $66 \mathrm{~mm}$.

\section{Sitta Amurensis, Swinh.}

Une paire d'Ara-Sambo et de Séoul, pris en janvier, parfaitement identiques aux oiseaux de l'Amour et du pays Oussourien.

\section{Troglodytes fumigatus dauricus (Tacz.).}

Trois mâles et deux femelles recueillis à Séoul en décembre et janvier.

Ces oiseaux de la Corée ressemblent beaucoup à ceux de Sidemi, quoique ils présentent une légère différence dans la rayure moins forte sur le devant du cou, qui, surtout dans un mâle et les deux femelles, ne dépasse pas la poitrine. Ils diffèrent encore plus sous ce rapport des oiseaux de la Daourie, dont le devant des parties inférieures du corps est fortement rayé en travers jusqu'à la gorge inclusivement. Il me paraît cependant que ce troglodyte continental diffère constamment du troglodyte japonais, dont je ne connais que deux exemplaires, un du Musée de St. Pétersbourg, provenant de Yokohama, l'autre du Musée de Varsovie, de l'ilôt Ascold. Ces deux troglodytes sont beaucoup plus roux en général que ceux du continent, à rayure très faible au dos, et n'ont point de raies foncées sur la gorge et sur toute la poitrine.

\section{Cinclus pallasi, Temm.}

Deux mâles et une femelle recueillis à Sambo Pielijan en mars et en décembre. Iris brun foncé.

\section{Accentor montanellus (Pall.).}

Un mâle et trois femelles de Séoul et d'Ara-Sambo, recueillis en décembre, janvier et février.

\section{Turdus fuscatus, Pall.}

Une paire de Séoul, recueillie en avril.

\section{Turdus naumanni, Temm.}

Deux mâles et deux femelles recueillis à Seoul en janvier et février.

Ces quatre exemplaires sont semblables à ceux qu'on trouve constamment dans le pays Oussourien, et que le Dr. Dybowski distingue sous le nom de T. abrekianus, tandis que $\mathrm{M}$. Radde les considère comme métis entre le $T$. naumanni et le T. ruficollis. Ces Grives présentent une telle rariabilité dans les différents détails de la coloration qu'il m'est impossible de comprendre la question. Les individus semblables à ceux de l'Oussouri et de la Corée se trouvent aussi dans les environs du Baical méridional, mais ils y sont rares, tandis que les deux espèces typiques citées y sont en grande majorité. $\mathrm{Au}$ contraire, dans une quarantaine d'individus que j'ai eu des différentes contrées du pays Oussourien et de la côte méridionale de ce pays, il n'y a en aucun individu typique du T. ruficollis ni du 
T. naumanni; le Dr. Dybowski assure aussi qu'il n'y a jamais vus de Grive typique de ces deux espèces, mais toujours des individus de cette variété ou race, et c'est la raison principale de son opinion que c'est une forme distincte, particulière à la contrée.

Ces oiseaux ont vraiment les caractères de la coloration intermédiaires entre ceux des deux espèces, mais les détails de la communauté sont très variables. Il $\mathrm{y}$ a des individus complètement unicolores sur les parties inférieures du corps comme chez le $T$. ruficollis typique, mais il y a des individus avec un mélange aussi fort de roux et le croupion uniformément roux comme chez le $T$. naumanni, le plus ty pique; entre ces deux modes de la coloration il y a des transitions en différents degrés.

Dans les uns de ces oiseaux le dessous depuis le menton jusqu'à la poitrine est comme dans les différentes variétés du $T$. ruficollis vrai, mais les côtés de l'abdomen sont aussi fort tachetés ou même plus comme dans le $T$. naumanni vrai.

Les uns ont la bande sourcilière aussi rousse comme celle du T. ruficollis, dans les autres elle est presque aussi blanche que celle du $T$. naumanni, dans les autres des transitions sans fin.

Tous les oiseaux de cette variété ont le sommet de la tête strié de foncé également comme dans les $\boldsymbol{T}$. ruficollis typiques, et jamais je n'ai-pas vus d'exemplaires à sommet de la tête aussi foncé comme dans le $T$. naumanni vrai.

Je n'ose pas de m'hasarder à trancher la question, et je la laisse à ceux qui pourront l'étudier en nature; mais je ne pus m'accorder avec l'opinion de $\mathbf{M}$. Radde, car je ne comprends pas quelle serait la raison pour que les métis de ces deux espèces se rassemblent dans cette contrée de l'extrême orient, évitée par les individus typiques des deux espèces. Tous le 7 exemplaires de Séoul ont le dessus du corps unicolore, avec un très petit mélange de roux au croupion; un a le sourcil comme celui du T. ruficollis, et aucun ne l'a pas blanc pur.

27. Oreocincla varia (Pall.).

Une paire recueillie en avril et mai à Séoul.

28. Monticola solitarius (Müll.).

Deux mâles pris à la fin de mai. Iris brun foncé.

29. Ruticilla aurorea (Pall.).

Trois mâles et une femelle recueillis à Séoul et à Soukamakoro en novembre, janvier, février et en juin.

30. Phyllopneuste borealis, Blas.

Une mâle tué à Séoul, en mai.

31. Phyllopneuste coronata (Temm.).

Une paire tuée à Séoul, en avril.

32. Phyllopneuste supciciliosa (Gm.).

Une mâle de Séoul. 


\section{Microscelis amaurotis (Less.).}

Dix exemplaires des deux sexes, recueillis aux environs de Séoul en décembre, janvier et février. Comparés au Musée de Varsovie avec les oiseaux de Yokohama.

\section{Motacilla leucopsis, Gould.}

Un mâle adulte en noces, tué à Séoul en avril.

\section{Motacilla ocularis, Swinh.}

Une paire en habit d'hiver complet de Séoul, recueillie en décembre.

36. Calobates melanope (Pall.).

Un mâle adulte tué en juin à Séoul.

37. Limonidromus indicus ( $\mathrm{Gm}$.).

Trois mâles de Séoul, recueillis en juin. Iris brun foncé.

\section{Alauda arvensis, L.}

Deux mâles, recueillis à Séoul en mars et en avril.

\section{Galerita cristata coreensis.}

Une paire recueillie à Séoul en janvier 1886. Comparés avec une grande série de races de Cochevis de différentes contrées de l'Europe, du nord de l'Afrique et de l'Asie centrale et occidentale.

Par la longueur du bec et par la coloration elle resemble le plus à la G. magna, Hume, du Turkestan (recueillis par Severtzoff), et en Mongolie occidentale aux environs du lac Lob-noor (recueillis par le général Prjewalski), mais s'en distingue par une taille moins forte, par le fond des parties supérieures du corps un peu plus foncé et plus roussâtre, sur lequel les taches brunes, centrales dans les plumes, sont en général plus grosses et plus fortement déssinées; le roux plus fort sur les tectrices supérieures de la queue; le brun noirâtre plus largement répandu le long du milieu des plumes de la huppe; en dessous la couleur jaunâtre distinctement plus forte; le roux du dessous de l'aile plus foncé et plus intense tirant sur le ton de cannelle ; la nuance des rectrices externes beaucoup plus foncée. La taille est moins forte.

La coloration est aussi semblable à celle de la G. macrorhyncha, Tristr., des plateaux sahariens de l'Algérie, mais elle s'en distingue aussi par les détails indiqués plus haut; la couleur de la rectrice externe est cependant plus rapprochée.

Elle ressemble aussi et plus encore par la grosseur et l'intensité des taches foncés dorsales à la G. abyssinica, Bp., de l'Egypte supérieur, ainsi que par la taille, mais elle est moins foncée en dessus à taches claires plus distinctes, les rectrices externes plus rousses, le cannelle du dessous de l'aile distinctement plus intense. La couleur générale des parties supérieures du corps, la grandeur et la force des taches foncés, ainsi que la nuance des rectrices externes la rapprochent le plus à la $G$. arenicola, Tristr., des plateaux élevés de l'Algérie (Constantine, Batna, \&c.), mais elle en diffère par le 
bec beaucoup plus long, les tectrices rousses de la queue à strie brune centrale foncée et grosse, tandis qu'elle est presque nulle chez l'oiseau cité ; le fond des parties inférieures et des côtés de la tête est beaucoup plus jaunâtre, au lieu de blanchâtre dans notre oiseau de la Corée. La deuxième rectrice présente la plus grande différence, elle n'a qu'une bordure roussâtre externe très fine, tandis que dans l'oiseau Algérien la plus grande moitié de la barbe externe est occupée par cette couleur et largement sur l'extrémité de toute la penne; sur les autres rectrices, sauf les médianes, il y a aussi des bordures terminales pareilles. La taille est presque la même.

Toutes les autres races qui me sont connues présentent des différences beaucoup plus grandes, la couleur des parties supérieures du corps est tout-à-fait autre.

o. Longueur totale 197 , vol 362 , aile 105 , queue 68 , bec 25 , tarse 14 , doigt médian 14 , ongle 4 , ongle du pouce 12 .

․ Longueur totale 185 , vol 330 , aile 94 , queue 62 , bec 23 , tarse 23 , doigt médian 17 , ongle 4 , ongle du pouce 11 .

o. Queue dépassant le bout des ailes de $25 \mathrm{~mm}$.

ㅇ. Queue dépassant le bout des ailes de $25 \mathrm{~mm}$.

40. Parus varius, Temm. et Schl.

Deux mâles et une femelle tués à Séoul le 8 février 1886 . Iris brun foncé.

41. Parus minor, Temm. et Schl.

Deux mâles recueillis à Séoul en novembre et en décembre. Semblables en tout aux oiseaux du pays Oussourien, moins verts et plus cendrés en dessus que les oiseaux du Japon.

\section{Pecilia palustris crassirostris, Tacz.}

Une femelle tuée à Séoul le 17 décembre. Parfaitement identique aux oiseaux du pays Oussourien.

43. Mecistura trivirgata (Temm. et Schl.).

Un exemplatre de Séoul.

\section{Suthora webbiana, Gr.}

Deux mâles et une femelle, recueillis à Séoul le 2 décembre. Comparés avec un exemplaire du Shensi méridional, provenant de la collection de l'Abbé David, et ne s'en distinguant que par la couleur du dos moins foncée et pas aussi nettement tranchée de la couleur rousse du cou postérieur. Ces oiseaux diffèrent plus des oiseaux de la race de la Mantchourie russe, à la quelles ils présentent une certaine transition.
o. Long. totale 141 , vol 168 , aile 52 , queue 67 millim.

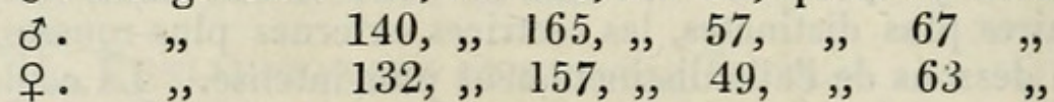

45. Oriolus diffusus, Sharpe.

Quatre mâles et une femelle de Séoul, recueillis en mai et en juillet. 
46. Lanius sphenocercus, Cab.

Deux mâles et une femelle adultes pris à Séoul et à Siongno en décembre et en février.

47. Phoneus bucephalus (Temm. et Schl.).

Un mâle pris à Séoul en mars.

48. Отоmela lucionensis (L.).

Quatre mâles recueillis à Séoul en mai et en juin.

49. Butalis latirostris, Raffl.

Un mâle tué à Séoul en mai.

50. Erythrosterna luteola (Pall.).

Un mâle tué à Séoul en mai.

51. Xanthopygia tricolor, Hartl.

Quatre mâles recueillis à Séoul, à la fin d'avril et en mai.

Egalement comme les oiseaux de la côte de la mer du Japan les uns ont le sourcil tout blanc, les autres en partie coloré légèrement de jaune.

52. Cyanoptila cyanomelena (Temm. et Schl.).

Une femelle de Séoul.

53. Pericrocotus cinereus, Lafr.

Deux mâles de Séoul, tués en mai.

54. Cyanopolius cyanus (Pall.).

Deux mâles et trois femelles de Tiumbudon, Siongno, Pedziuumne, recueillis en janvier et mars.

55. Garrulus brandti, Eversm.

Une paire de Séoul et de Tempunkudzi, tués en janvier et mars.

Semblables en tout aux oiseaux Oussouriens.

56. Pica caudata Japonica (Temm. et Schl.).

Deux mâles, deux femelles et un jeune, tués en décembre et en janvier.

Semblables en tout aux oiseaux du pays Oussourien.

57. Monedula daurica (Pall.).

Une paire de Siongno, recueillie en mars.

58. Corvus macrokhynchus Japonensis (Bp.).

Deux femelles tuées à Séoul en janvier.

59. Frugilegus pastinator (Gould).

Un exemplaire de Pedziu-umne pris en mars.

Proc. Zool. Soc.-1887, No. XL. 
60. Nucifraga caryocatactes, L.

Un exemplaire pris à Séoul en novembre.

61. Sturnus cineraceus, Temm. et Schl.

Une mâle pris à Séoul en avril. Iris brun foncé.

62. Emberiza fucata, Pall.

Un mâle pris en mai à Séoul.

63. Emberiza cioides, Brandt.

Un mâle et deux femelles pris en décembre à Séoul.

64. Emberiza spodocephala, Pall.

Un mâle pris en mai à Séoul.

65. Emberiza elegans, Temm.

Trois mâles de Séoul et d'Ara-Sambo, pris en décembre, en janvier et en mars.

66. Schenicola polaris (Midd.).

Trois mâles et une femelle pris à Siongno en mars.

67. Passer montanus (L.).

Une paire, prise à Séoul en novembre et décembre.

68. Fringilla montifringilla, L.

Deux mâles et une femelle, pris à Séoul, à Ara-Sambo et à Tempunkudzi, en décembre.

69. Chlorospiza sinica (L.).

Un mâle pris à Séoul en mars.

70. Chrysomitris spinus (L.).

Quatre mâles et cinq femelles, pris à Séoul en décembre et en avril.

Sur ces quatre mâles il y a trois dont le devant du cou est fort coloré d'une nuance orangé roussâtre, cette couleur est répandue dans un de ces exemplaires sur toute la poitrine, sur les côtés de la tête et sur toute la bande sourcilière, dans l'autre elle ne dépasse pas la région jugulaire et est peu répandue sur les côtés de la tête, dans le troisième elle est moins forte et moins disposée.

71. Coccothraustes vulgaris Japonicus, Temm. et Schl. Une femelle, prise à Séoul en avril. Iris et bec d'un bleu pâle.

72. Propasser roseus (Pall.).

Un mâle adulte, pris en février à Séoul.

73. Uragus Sanguinolentus (Te mm. et Schl.).

Trois femelles de Usambo et de Kuksude-i, prises en décembre. 


\section{Loxia curvirostra albiventris (Swinh.).}

Cinq mâles et deux femelles pris à Séoul en février.

Ces oiseaux de la Corée se distinguent des oiseaux de la Sibérie et de l'Europe centrale par une taille un peu moins forte et par la couleur rouge du mâle, qui est carminée dans ces oiseaux de la Corée, au lieu de ponce sanguiné des Becs-croisés communs. Quant à la couleur blanche du milieu du ventre, elle n'est pas plus répandue dans ces oiseaux coréens que dans les Becs-croisés sibériens et européens. Tous les Becs-croisés que j'ai eu des différents localités de la Sibérie orientale sont identiques aux oiseaux européens.

Dimensions des oiseaux de la Corée :-

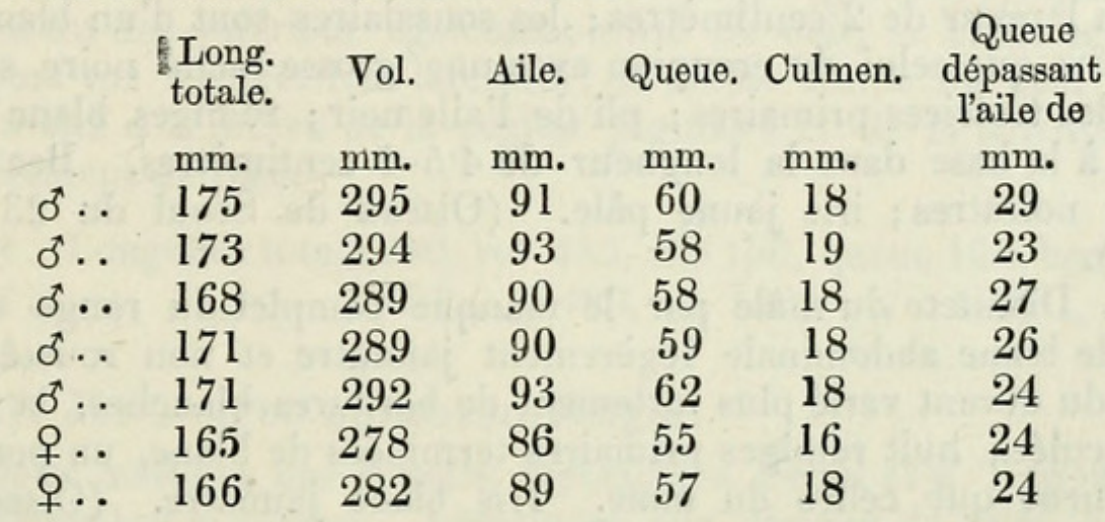

75. Pyrrhula orientalis, Temm. et Schl.

Une paire de Séoul, prise en février et mars.

\section{Cuculus canorus telephonus (Heine).}

Un mâle tué en novembre à Séoul au commencement d'avril.

\section{Gecinus canus (Gm.).}

Une femelle tuée en novembre à Séoul. Cet oiseau est beaucoup plus vert au dos que la plus grande pluralité de ces Pics du pays Oussourien.

78. Thriponax KalinowskiI, sp. $\mathrm{n}$.

Th. nigerrimus; dorso infero cum uropygio abdominequo favidoalbis, regione anali nigra, albido undulata; tibiis albo nigroque variis, remigibus primariis albo terminatis; subalaribus abdomini concoloribus; collo antico supero tenuissime albo vario.

Mas pileo toto fasciaque malari vivide cinnabarino-rubris.

Fem. capite toto nigro.

$\sigma^{*}$ ad. Plumage général noir intense, à dos inférieur et le croupion d'un blanc légèrement jaunâtre ; abdomen fort coloré de roussâtre, assez clair sur une grande majorité de plumes, d'un ocreux sale sur les autres ${ }^{1}$, tout le sommet de la tête jusqu'à la nuque inclusivement est d'un beau rouge vermillon trés vif, bande malaire étroite d'un rouge plus obscur ne dépassant pas le niveau du bord postérieur de l'œil ;

1 Cette couleur rousse paraît être superficielle, ce qui a souvent lieu dans le plumage avant la mue chez beaucoup d'autres Pics. 
plumes de la gorge noirâtres bordées de blanchâtre, quelques petites macules ou bordures blanches sur le noir du haut du devant du cou, une raie composée de petites taches blanches située le long des côtés du cou commençant au niveau du milieu de l'œil, une tache composée de macules ou de petites stries blanches derrière l'orcille; région anale noirâtre rayée de blanchâtre par les bordures des plumes; plumage des jambes varié de noirâtre et de blanchâtre. Cinq rémiges primaires terminées de blanc, sur la première abortive il n'y a qu'une petite macule terminale, tandis que sur la troisième la barbe externe blanche dans la longueur de $2 \cdot 5$ centimètres, l'interne dans plus d'un centimètre; le blanc terminal de la quatrième n'atteint pas la largeur de 2 centimètres; les sousalaires sont d'un blanc plus jaunâtre que celui du croupion avec une grosse tache noire sur les grandes tectrices primaires; pli de l'aile noir ; rémiges blanc jaunâtres à la base dans la longueur de $4 \cdot 5-5$ centimètres. Bec noir ; pieds noirâtres; iris jaune pâle. (Oiseau de Séoul du 23 juin, 1886).

․ Distincte du mâle par le manque complet du rouge sur la tête, le blanc abdominale légèrement jaunâtre et non roussâtre, le haut du devant varié plus fortement de bordures blanches, la gorge immaculée; huit rémiges primaires terminées de blanc, un peu plus largement que celles du mâle. Iris blanc jaunâtre. (Oiseau de Siongno tué le 28 février, en plumage frais.)

La couleur blanche est fort répandue sur la barbe interne des rémiges primaires, dans la $1^{\text {re }}$ et $2^{\mathrm{e}}$ rémiges le blanc dépasse le tiers basal de ces rémiges, dans la $4^{\mathrm{e}}$ il dépasse le quart basal; dans les rémiges secondaires le blanc est aussi largement disposé; la baguette de toutes ces rémiges est aussi blanche dans sa partie basale.

đ. Longueur totale 495 , vol 775 , aile 246 , queue 173 , bec de la commissure 66 , tarse 31 , doigt antérieur externe 28 , ongle en diamètre 18 , doigt postérieur externe 18 , ongle en diamètre 16 , queue dépassant le bout des ailes de $90 \mathrm{~mm}$.

ㅇ. Longueur totale 453 , vol 786 , aile 252 , queue?, bec 69 , tarse 32 , doigt antérieur ext. 28 , ongle 19, doigt postérieur externe 19, ongle $17 \mathrm{~mm}$.

\section{Picus major, $\mathbf{L}$.}

Une paire de Séoul, prise en février. Sous le rapport de la forme du bec et la coloration semblables à ceux de l'Europe centrale, de la Daourie et du Baical méridional, ils ont cependant le blanc moins prolongé sur le devant des scapulaires, les bandes blanches un peu moins larges sur les rémiges. Le blanc et le noir également disposé sur les rectrices, mais à bandes noires distinctement plus larges. Le mâle a le blanc du dessous sali de grisâtre, le front également sale, la tache auriculaire et le devant du cou colorés légèrement de roussâtre sale. Le plumage du dessous de la femelle plus pur, mais coloré fortement de la nuance de cuire tannée sur la tache auriculaire et sur tout l'abdomen.

ð. Longueur totale 247 , vol 430 , aile 130 , queue 85 , bec 33 , tarse 22, doigt ext. ant. 15, ongle $10 \mathrm{~mm}$. 
ㅇ. Longueur totale 250 , vol 423 , aile 133 , queue 90 , bec 30 , tarse 22 , doigt ext. ant. 14 , ongle 10 .

Queue dépassant le bout des ailes de 38 millim. chez le ${ }^{\star}$, de 45 chez la 오.

\section{Picus leuconotus, Bechst.}

Deux mâles de Séoul, pris en décembre et en février. Ces oiseaux ne présentent aucune différence de ceux de l'Europe centrale, ils ont les bandes blanches transalaires également larges, les stries du dessous de la même grosseur, la couleur rouge également répandue, le blanc du dos inférieur également varié de noir, le blanc également disposé sur les rectrices latérales; la même forme du bec. Un de ces mâles a le milieu de la région jugulaire et de la poitrine coloré légèrement de rosé.

o. Longueur totale 290 , vol 485 , aile 150 , queue 100 , bec $45 \mathrm{~mm}$. o. " $\quad 294,, 490,, 150$, , $102,, 47 \mathrm{~mm}$.

\section{Jungipicus dör riesi, Hargitt.}

Deux mâles et une femelle, recueillis à Séoul et à Tempunkudzi, en décembre et février. Identiques aux oiseaux de l'ilôt Ascold et de la côte de la Manchourie Russe.

\section{Jungipicus SeEbohmi, Hargitt.}

Une mâle de Séoul.

Semblable en tout aux oiseaux typiques de Yokohama de la collection de M. Hargitt, et aux oiseaux de la Manchourie Russe, mais cet exemplaire est d'une taille un peu moins forte. Longueur de l'aile $83 \mathrm{~mm}$., queue $50 \mathrm{~mm}$.

\section{Columba rupestris (Pall.).}

Une femelle tuée à Séoul en avril.

84. Turtur rupicola (Pall.).

Une jeune femelle tuée à Séoul en décembre.

\section{Turtur torquatus, Briss.}

Un mâle et trois femelles recueillis à Séoul en novembre, décembre et mai.

\section{Bonasia betulina, Dresser.}

Deux mâles et une femelle recueillis à Sambo Pielijan en norembre et décembre.

Les mâles ne présentent aucune différence de la grande pluralité des gélinottes de l'Europe centrale et du pays Oussourien; la femelle présente une anomalie dans sa gorge noire comme celle du mâle, mais mélangée légèrement de fauve et le menton blanchâtre. 
87. Coturnix communis, Bonnat.

Trois mâles et deux femelles de Sesulma, Siongno, Ara-Sambo et C.-chonmal, recueillis en novembre, janvier et mars.

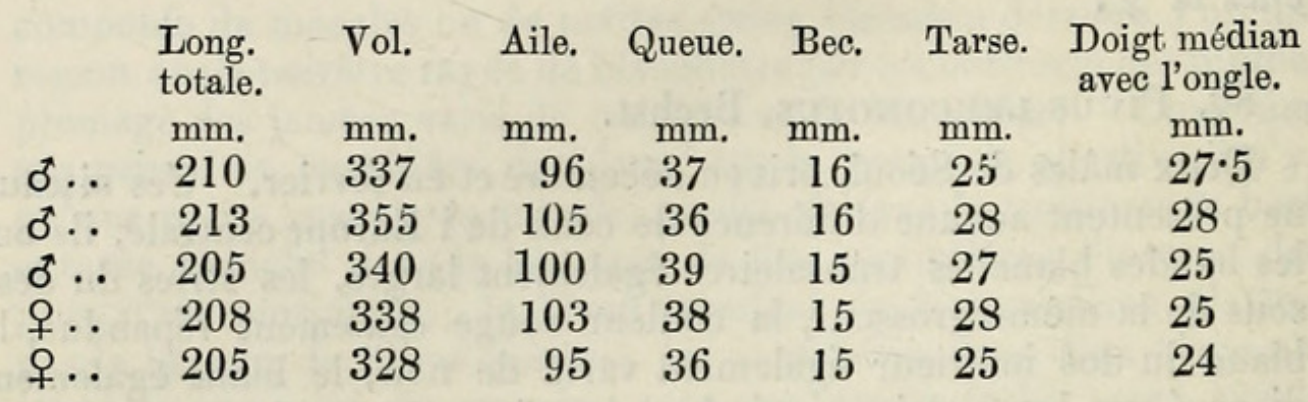

88. Phasianus torquatus, Temm.

Une paire prise aux environs de Séoul en janvier. Coloration en général considérablement plus obscure que celle des Faisans du pays Oussourien. Le mâle a toutes les couleurs plus foncées, ce qui est le plus frappant au bas du cou (auchenium), où elle est d'un roux doré au lieu de straminé ; le noir du milieu des parties inférieures du corps nrolongé jusque sur la poitrine, le vert au cou passant moins au bleu; la couleur de la poitrine, de la région jugulaire et des flancs beaucoup plus foncée, etc.; il lui manque de macule blanche au dessous des oreilles, mais la plus grande différence est dans la forme du collier blanc, qui est presque d'égale largeur partout, tandis que celui du Faisan Oussourien est fort attenué en arrière et élargi sur les côtés ; le blanc de la bande sourcilière n'atteint pas la base des huppes latérales, tandis qu'il la dépasse chez l'oiseau cité. Jugeant de la description dans l'ourrage sur 'Les Oiseaux de la Chine' de l'Abbé David et M. Oustalet, ce Faisan Coréen est différent dans les différents détails de toutes les trois variétés locales mentionnées dans ce travail. La femelle est aussi en général considérablement plus foncée.

89. \#gialitis minor (Mey. et Wolf).

Deux mâles et deux femelles recueillis à Séoul en avril.

90. Ægialitis placidus (Gr.).

Un mâle de Séoul, tué en janvier.

91. Vaneluus cristatus, Mey. et Wolf.

Une femelle tuée en mars à Dultoni.

92. Tringa cinclus, L.

Un mâle tué à Séoul en novembre.

93. Tringa minuta, Leis].

Deux paires d'oiseaux jeunes tués en novembre.

94. Gallinago scolopacina, Bp.

Un exemplaire de Séoul tué en novembre. 
95. Gallinago stenura, Kuhl.

Un exemplaire de Séoul tué en novembre.

96. Grus viridirostris, Vieill.

Une paire de Sesulma et de Piengga; yeux presque noirs.

97. Grus leucauchen, Temm.

Deux femelles de Séoul et de Dultoni recueillis en mars. Iris jaune rougeâtre.

98. Ibis nippon, Temm.

Trois mâles et une femelle de Séoul et de Gouran, tués en décembre et janvier.

99. Ibis nippon Sinensis, Oust.

Une femelle tuée à Tongdje en mai.

100. Ciconia boyciana, Swinh.

Mâle adulte tué en mars. Bec noirâtre ; pieds d'un rouge obscur ; tour dénué de l'œil et peau gulaire rouge, iris jaune pâle.

Longueur totale 1330 , vol 2470 , aile 705 , queue 256 , bec 288 , tarse 265 , partie dénuée des tibiæ 186 , doigt médian 98 , aile dépassant la quéue de $10 \mathrm{~mm}$.

101. Ardea cinerea, L.

Un mâle tué à Séoul en avril.

102. Ardea alba modesta (J. E. Gr.).

Un mâle tué à Séoul en juillet. Iris jaıne pâle; bec et pieds noirs. Longueur totale 993 , vol 1460 , aile 370 , queue 135 , bec 140 , tarse 190 , partie dénuée des tibiæ 105 , doigt médian 97 , ongle 10 , scapulaire dépassant le bout de l'aile $144 \mathrm{~mm}$.

103. Gallicrex cinerea (Gm.).

Femelle tuée à Séoul en juillet.

104. Sternula sinensis (Gm.).

Six mâles et deux femelles, tués à Séoul en mai et juin.

105. Cairina rutila (Pall.).

Un mâle tué à Seoul en janvier.

106. Clangula glaucion (L.).

Une paire recueillie à Gouran en décembre.

107. Oidemia americana, Sw. et Rich.

Une femelle de Gouran (port Lazarew), tué en décembre. Iris presque noir. 


\section{$2 \mathrm{BHL}$ Biodiversity Heritage Library}

1887. "Liste des Oiseaux recueillis en Coree par M. Jean Kalinowski." Proceedings of the Zoological Society of London 1887, 596-611. https://doi.org/10.1111/j.1469-7998.1887.tb08151.x.

View This Item Online: https://www.biodiversitylibrary.org/item/99199

DOI: https://doi.org/10.1111/j.1469-7998.1887.tb08151.x

Permalink: https://www.biodiversitylibrary.org/partpdf/70217

\section{Holding Institution}

Natural History Museum Library, London

\section{Sponsored by}

Natural History Museum Library, London

\section{Copyright \& Reuse}

Copyright Status: Public domain. The BHL considers that this work is no longer under copyright protection.

This document was created from content at the Biodiversity Heritage Library, the world's largest open access digital library for biodiversity literature and archives. Visit BHL at https://www.biodiversitylibrary.org. 